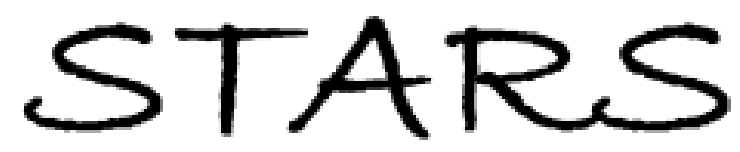

University of Central Florida

STARS

$1-1-2015$

\title{
The perturbation energy: A missing key to understand the "nobleness" of bulk gold
}

\author{
Marisol Alcántara Ortigoza \\ University of Central Florida \\ Sergey Stolbov \\ University of Central Florida
}

Find similar works at: https://stars.library.ucf.edu/facultybib2010 University of Central Florida Libraries http://library.ucf.edu

This Article is brought to you for free and open access by the Faculty Bibliography at STARS. It has been accepted for inclusion in Faculty Bibliography 2010 s by an authorized administrator of STARS. For more information, please contactSTARS@ucf.edu.

\section{Recommended Citation}

Ortigoza, Marisol Alcántara and Stolbov, Sergey, "The perturbation energy: A missing key to understand the "nobleness" of bulk gold" (2015). Faculty Bibliography 2010s. 6734.

https://stars.library.ucf.edu/facultybib2010/6734

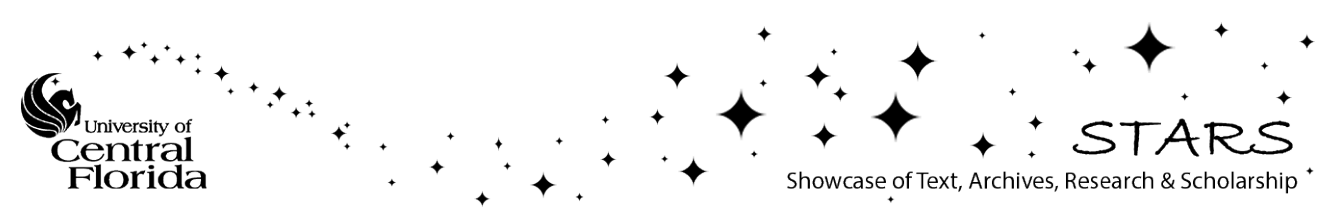




\section{The perturbation energy: A missing key to understand the "nobleness" of bulk gold}

Cite as: J. Chem. Phys. 142, 194705 (2015); https://doi.org/10.1063/1.4919774

Submitted: 04 October 2014 . Accepted: 24 April 2015 . Published Online: 19 May 2015

Marisol Alcántara Ortigoza, and Sergey Stolbov
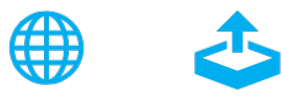

\section{ARTICLES YOU MAY BE INTERESTED IN}

A machine learning approach to graph-theoretical cluster expansions of the energy of adsorbate layers

The Journal of Chemical Physics 147, 054106 (2017); https://doi.org/10.1063/1.4985890

A consistent and accurate ab initio parametrization of density functional dispersion correction (DFT-D) for the 94 elements $\mathrm{H}-\mathrm{Pu}$

The Journal of Chemical Physics 132, 154104 (2010); https://doi.org/10.1063/1.3382344

Modification of the surface electronic and chemical properties of $\mathrm{Pt}(111)$ by subsurface $3 \mathrm{~d}$ transition metals

The Journal of Chemical Physics 120, 10240 (2004); https://doi.org/10.1063/1.1737365

Where in the world is AIP Publishing?

Find out where we are exhibiting next 


\title{
The perturbation energy: A missing key to understand the "nobleness" of bulk gold
}

\author{
Marisol Alcántara Ortigoza ${ }^{\mathrm{a})}$ and Sergey Stolbov ${ }^{\mathrm{b})}$ \\ Department of Physics, University of Central Florida, Orlando, Florida 32816, USA
}

(Received 4 October 2014; accepted 24 April 2015; published online 19 May 2015)

\begin{abstract}
The nobleness of gold surfaces has been appreciated since long before the beginning of recorded history. Yet, the origin of this phenomenon remains open because the so far existing explanations either incorrectly imply that silver should be the noblest metal or would fail to predict the dissolution of Au in aqua regia. Here, based on our analyses of oxygen adsorption, we advance that bulk gold's unique resistance to oxidation is traced to the large energy cost associated with the perturbation its surfaces undergo upon adsorption of highly electronegative species. This fact is related to the almost totally filled $d$-band of Au and relativistic effects, but does not imply that the strength of the adsorbate-Au bond is weak. The magnitude of the structural and charge-density perturbation energy upon adsorption of atomic oxygen - which is largest for $\mathrm{Au}$ - is assessed from first-principles calculations and confirmed via a multiple regression analysis of the binding energy of oxygen on metal surfaces. @ 2015 AIP Publishing LLC. [http://dx.doi.org/10.1063/1.4919774]
\end{abstract}

\section{INTRODUCTION}

It is well known that gold forms very stable bulk alloys, oxoaurates are now possible to synthesize, ${ }^{1}$ and gold ions, clusters, as well as overlayers have a very reach chemistry and catalytic applications. ${ }^{2-4}$ Nevertheless, it is also well known that the surfaces of bulk Au embody unparalleled cases of metal resistance towards forming compounds with highly electronegative atoms such as $\mathrm{O}, \mathrm{N}$, and $\mathrm{C}$. The prime and simplest indication of the latter is undoubtedly the binding of atomic oxygen on metal surfaces. ${ }^{5}$ In this regard, adsorption calorimetry and temperature-programmed desorption measurements ${ }^{6-15}$ have confirmed that, among all transition metal surfaces, $\mathrm{Au}(111)$ forms the least stable bond with atomic oxygen. Hammer and Nørskov, however, arrived at a widely accepted explanation for $\mathrm{Au}$ inertness by considering the $\mathrm{H}_{2}$ dissociation reaction on $\mathrm{Au}, \mathrm{Cu}, \mathrm{Ni}$, and $\mathrm{Pt}$. The central idea was that $\mathrm{Au}$ binds $\mathrm{H}$ weaker than do $\mathrm{Pt}$ and $\mathrm{Ni}$ because the antibonding $d$-states of Au are essentially filled. ${ }^{16}$ The nobleness of gold has also been attached to the relatively short radius of the $6 s$ and $6 p$ orbitals of Au resulting from the large relativistic effects in Au. ${ }^{2,19}$ The $6 s$ and $6 p$ orbitals of Au therefore screen better the nucleus, which makes their work function high. The high work function of $\mathrm{Au}$ and $\mathrm{Pt}$ also explains their dissolution resistance in some acids. Nevertheless, since some Pt-group elements, as well as some early transition metals, are somewhat resistant to aqua regia, a relativistic-effects-based explanation would fail to predict that $\mathrm{Au}$ and $\mathrm{Pt}$ readily dissolve in it. Furthermore, there is another, and contradictory aspect of the relativistic effects. The $6 s$ and $6 p$ orbitals screen better the nucleus at the expense of destabilizing and expanding the $5 d$ orbitals. Relativistic effects thus enlarge the radius of the $5 d$ orbitals, hence affording stronger covalent bonds for $\mathrm{Au}$ than for $\mathrm{Ag}$.

\footnotetext{
a) Electronic address: Marisol.AlcantaraOrtigoza@ucf.edu

b) Electronic address: Sergey.Stolbov@ucf.edu
}

This effect should then render Au less noble, which in fact is in line with the predictions of the $d$-band model, as we shall see.

The central idea of Hammer and Nørskov to explain Au nobleness in terms of the filling of the $d$-band and the characteristic radius of the atomic orbitals $\left(r_{d}\right)$ later set the ground for the so-called $d$-band-center model. The significance of the $d$-band model lies in the possibility to predict the binding energy of adsorbates whenever the covalent bond is the foremost or the only variable (from metal to metal) contribution to the binding energy (see Ref. 17 and the Appendix). The perspective given by the $d$-band model has been applied to understand the catalytic activity of a number of single-element and bimetallic $^{20,21}$ transition-metal surfaces ${ }^{5,17,22}$ and has provided an essential tool for designing catalysts. ${ }^{4,22}$ Nonetheless, there are critical inconsistencies between its predictions and experiment and/or $a b$ initio calculations that involve adsorption on group11 and group-10 metal surfaces. Below, we only focus on the cases relevant to the subject of this work. Additional cases can be found in the Appendix.

Failures in rationalizing the metal-O bond strength by the filling degree of the metal d-band and the characteristic atomic radius $r_{d}$. First of all, the seminal argument that $\mathrm{Au}$ binds adsorbates relatively weak because Au antibonding $d$ states are nearly fully occupied completely fails when we turn to the other metals of the same group, $\mathrm{Ag}$ and $\mathrm{Cu}$. For instance, although the $d$-band of $\mathrm{Ag}$ and $\mathrm{Cu}$ is also almost entirely filled (see Fig. 1), (1) the binding energy of the $\mathrm{OH}$ molecule on $\mathrm{Ag}(111)$ is as large as that on $\mathrm{Pt}(111) .{ }^{24}(2)$ More strikingly, the experimental heat of adsorption of $\mathrm{O}_{2}$ upon dissociation $\left(H_{\text {ads }}^{D}\right.$ ) on $\mathrm{Au}(111)$ is $\sim 30 \mathrm{kcal} / \mathrm{O}_{2}$ smaller than that on $\mathrm{Ag}(111)$ and up to $\sim 100 \mathrm{kcal} / \mathrm{O}_{2}$ smaller than that on $\mathrm{Cu}(111) .{ }^{6-9}$ While the unexpected difference in reactivity between $\mathrm{Au}$ and $\mathrm{Ag}$ remains unclear, the explanation for the high reactivity of $\mathrm{Cu}$ is not satisfactory. The fact that $\mathrm{Cu}$ is more reactive than $\mathrm{Au}$ has been traced to the repulsive interaction between metal-adsorbate that takes place when their non-orthogonal 


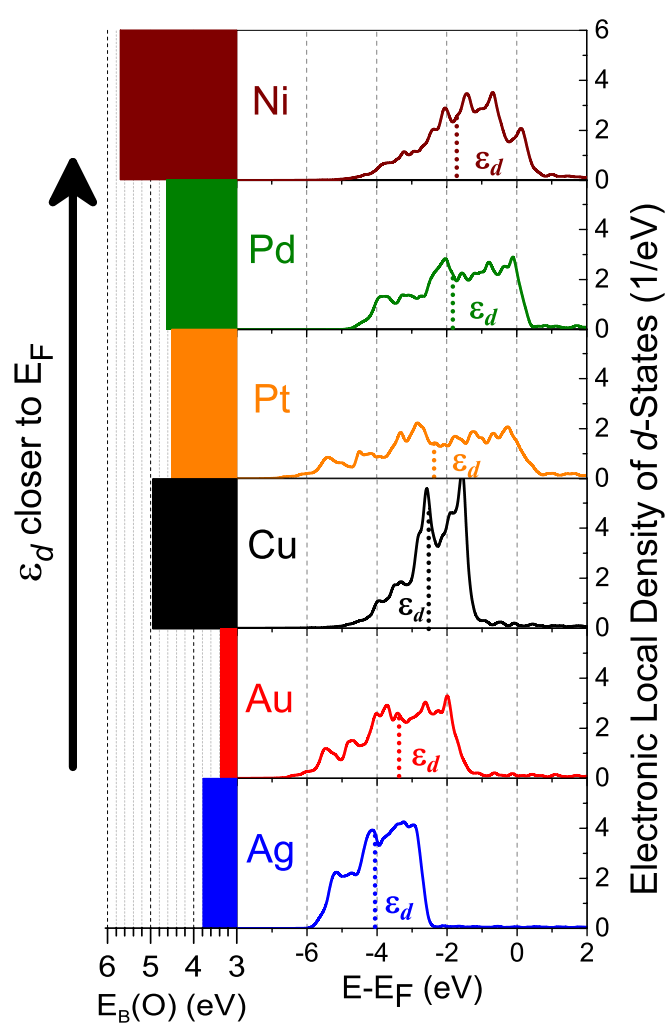

FIG. 1. (Left) Calculated binding energy of oxygen $\left(E_{B}(O)\right)$ on various transition metals at the lowest-energy ( $\mathrm{fcc} / \mathrm{hcp}$ ) hollow site. The arrow indicates that the elements are ordered from bottom to top according to the position of their $d$-band center $\left(\epsilon_{d}\right)$ with respect to the Fermi level. (Right) Density of electronic $d$-states of the metal surface atoms (number of electronic states per atom per $\mathrm{eV}$ ). The dotted line indicates the $d$-band center.

electronic states overlap (see Ref. 16 and the Appendix). This orthogonalization energy "cost" has been incorrectly assumed larger for $\mathrm{Au}$ because the characteristic radius $\left(r_{d}\right)$ of $\mathrm{Au}$ $d$-orbitals is $50 \%$ larger than that of $\mathrm{Cu}^{16,25}$ Such rationale is not valid because the covalent-bond energy $E_{d-h y b}$ as a whole (including both hybridization and orthogonalization terms) is directly proportional to the interatomic matrix element for the metal-adsorbate bond $V_{s d}^{2}=C r_{d}^{3} / a_{0}^{7}$, where $a_{0}$ is the metaladsorbate bond-length and $C$ is a constant (see the Appendix). Of course, one could still argue that $\mathrm{Cu}$ is more reactive than $\mathrm{Au}$ because $a_{0}$ is normally shorter for $\mathrm{Cu}$ than for $\mathrm{Au}$ (see Table II) and is raised to a higher power than $r_{d}$ in the expression for $V_{s d}^{2}$. Nevertheless, such assumption turns out to be incorrect because $V_{s d}^{2}$ for the $\mathrm{Au}-\mathrm{O}$ bond is actually $50 \%$ larger than that for the $\mathrm{Cu}-\mathrm{O}$ bond. Finally, in addition to the fact that $E_{d-h y b}$ is not necessarily the decisive contribution to the binding energy of an adsorbate, assuming that the occupation number of the $d$-orbitals of group-11 metal surfaces is equal to 10 is a poor approximation that can be improved when applying the $d$-band model. It is well documented that $\mathrm{Au}-\mathrm{Au} s-p-d$ hybridization creates unoccupied $d$-states. ${ }^{26-29}$

Failures in rationalizing the metal-O bond strength by the energy of the d-band center. Based on the dependence of $E_{d-h y b}$ on the energy of the $d$-band center, one could try to justify qualitatively that $\mathrm{Cu}$ is more reactive than $\mathrm{Au}$ on the grounds of the $d$-band model, since $\epsilon_{d}$ is closer to $\mathrm{E}_{F}$ for $\mathrm{Cu}$. Nevertheless, we shall see later that not even this fact makes the $\mathrm{Cu}-\mathrm{O}$ bond more covalent than the $\mathrm{Au}-\mathrm{O}$ bond. Furthermore, within the $d$-band model not only should Ag be equally inert as Au but even much more inert; namely, $\epsilon_{d}$ of $\operatorname{Ag}(111)$ is significantly deeper than that of $\mathrm{Au}(111)$ (not to mention that $V_{s d}$ is also smaller for $\mathrm{Ag}-\mathrm{O}$ than for $\mathrm{Au}-\mathrm{O})$.

Here, we shall: (1) draw attention to the fact that the $d$ band model may correctly predict the energy of covalent bonds and yet fails to describe the overall bond strength (see the Appendix); (2) demonstrate that the relativistic effects are not sufficient to explain the remarkably small heat of adsorption of oxygen; and finally and most importantly, (3) provide evidence that the strong perturbation to the $\mathrm{Au}-\mathrm{Au}$ bonds brought in through the Au-adsorbate bond is a key factor leading to gold nobleness.

\section{COMPUTATIONAL DETAILS}

Our first-principles calculations are performed with the Vienna $a b$ initio simulation package (VASP) ${ }^{31}$ using projector-augmented-wave-method ${ }^{32}$ pseudopotentials. ${ }^{33}$ The pseudopotentials of $\mathrm{Pt}, \mathrm{Au}$, and $\mathrm{Ir}$ include the scalar relativistic effects (mass-velocity and Darwin terms). We treat the exchange-correlation functional in the generalized gradient approximation using the functional by Perdew-BurkeErnzerhof. ${ }^{34}$ The maximum kinetic energy of the plane waves used to describe valence states is set to $500 \mathrm{eV}$. The (111) surfaces of nine metals were modeled using a 9-layer slab of a $(2 \times 2)$ in-plane periodicity with one $\mathrm{O}$ atom on the surface at the lowest-energy (fcc/hcp) hollow site. For the three group11 metals and $\mathrm{Pt}$, the results were confirmed by using slabs of a $(3 \times 3)$ in-plane periodicity. Notice that one could think that not considering the well-known $22 \times \sqrt{3}$ of $\mathrm{Au}(111)$ may have biased our results. This can be cast aside on the grounds that the herringbone reconstruction has a large length-scale $(\sim 6 \mathrm{~nm})$, for which unreconstructed terraces predominate. Moreover, DFT calculations indicate that the energy reduction upon reconstruction is only $\sim 6 \mathrm{meV}$ per atom $(\sim 25 \mathrm{meV}$ in our $2 \times 2$ supercell). ${ }^{35}$

For the in-plane periodicity of clean and adsorbed surfaces, the optimized lattice parameter of the corresponding bulk metal, obtained from our calculations, is used. In all slab calculations, a vacuum space of $14 \AA$ separates the periodic images of the slab to avoid interaction between them. Integrations inside the Brillouin zone are performed over a kpoint mesh determined by the scheme proposed by MonkhorstPack. ${ }^{36}$ The k-point meshes used are $16 \times 16 \times 16,8 \times 8 \times 1$, and $6 \times 6 \times 1$ k-point meshes for bulk, $2 \times 2$-surface, and $3 \times 3$-surface calculations, respectively.

For convergence of the total energy, an energy cutoff of $5 \times 10^{-6} \mathrm{eV}$ is enforced. The ground-state geometry of each system is achieved by reducing the Hellmann-Feynman forces $^{33}$ below $3 \times 10^{-3} \mathrm{eV} / \AA$. Moreover, in calculating ground-state geometries, each atom in the corresponding 9-layer slab has been relaxed applying the above-mentioned criterion. Changes in the total energy at the end of the relaxation were smaller than $1 \times 10^{-5} \mathrm{eV}$. This parameters allowed us to calculate the force constants reliably for displacements as small as $0.005 \AA$. The metal-O charge transfer is obtained via the Bader analysis method. ${ }^{37}$ We obtain real-space force constants, vibrational frequencies, and eigenvectors 
of $\mathrm{O}$ on $\mathrm{Au}(111)$ and $\mathrm{Ag}(111)$ with the finite-displacement method. The surface atoms are kept fixed because the force constant of the metal-metal bonds are irrelevant and, furthermore, to avoid the coupling of the metal-O and the metal-metal force constants. To visualize the charge density profiles, we use the XCrySDen software. ${ }^{38}$

The projected local densities of states are obtained by projecting the wave functions (plane waves) onto spherical harmonics followed by integrating over Wigner-Seitz spheres. The latter is done using the Gaussian smearing method and the width of the smearing for the chosen k-point mesh is $0.1 \mathrm{eV}$. The radius of the Wigner-Seitz spheres was varied from values for which the spheres do not overlap to values for which they overlap (containing in this way the total charge of the system) in order to ascertain that no significant dependence exists on this parameter.

The adsorption energy of oxygen is obtained in terms of the total energy of (1) the totally relaxed O-metal system $\left(E_{\text {total }}^{R}(O /\right.$ metal $\left.)\right),(2)$ the relaxed clean metal substrate $\left(E_{\text {total }}^{R}(\right.$ metal $\left.)\right)$, and (3) atomic oxygen $\left(E_{\text {total }}(O)\right) . E_{B}(O)$ is thus obtained as $E_{B}(O)=\mid E_{\text {total }}(O /$ metal $)-E_{\text {total }}^{R}($ metal $)$ $-E_{\text {total }}(O) \mid$. Note that the above equation does not take into account the zero-point energies (ZPE). However, our calculations for several metals substrates show that changes in ZPE do not change $E_{B}(O)$ by more than few tens of meV. Notice also that $E_{B}(O)$ is considered positive and, therefore, factors that weaken the bond are considered to reduce $E_{B}(O)$ and thus correspond to negative numbers, whereas factors that strengthen the bond are considered to increases $E_{B}(O)$, and thus correspond to positive numbers.

\section{RESULTS}

\section{A. $O$ binding energy and ionicity of the metal-O bond}

The applicability limits of the $d$-band model exposed by the experimental investigations mentioned in the introduction are also well documented by Density-FunctionalTheory (DFT) calculations. In particular, as shown in Table II, $a b$ initio calculations correctly simulate gold's inertness since the calculated $E_{B}(O)$ (as defined in Sec. II) on $\mathrm{Au}(111)$ is substantially smaller than on $\mathrm{Ag}(111)$ and $\mathrm{Cu}(111)$ (The optimized lattice parameters of the metal surfaces are shown in Table I). Nonetheless, having acknowledged that the $d$ band model aims to capture only the contribution to $E_{B}(\mathrm{O})$ from the hybridization between the electronic states of the adsorbate and the $d$-states of metals, the above results should not be all surprising; namely, $\mathrm{O}$ is highly electronegative and, therefore, the metal-O bond is not necessarily predominantly covalent but also partially ionic. In fact, even though the measured energy of the $1 s$-state of atomic $\mathrm{O}$ hardly changes

TABLE I. Lattice parameter(s) of the metals in the bulk.

\begin{tabular}{lccccc}
\hline \hline & $a(\AA)$ & & $a(\AA)$ & & $a(\AA)$ \\
\hline $\mathrm{Cu}$ & 3.636 & $\mathrm{Pd}$ & 3.960 & $\mathrm{Ru}$ & $2.731(c / a=1.580)$ \\
$\mathrm{Ag}$ & 4.164 & $\mathrm{Ni}$ & 3.517 & $\mathrm{Ir}$ & 3.877 \\
$\mathrm{Au}$ & 4.173 & $\mathrm{Pt}$ & 3.977 & $\mathrm{Rh}$ & 3.850 \\
\hline \hline
\end{tabular}

upon adsorption, adsorption calorimetry, and temperatureprogrammed desorption measurements show that there is a net repulsion between adsorbed $\mathrm{O}$ atoms and it is also well known that $\mathrm{O}$ adsorption induces electric dipoles at the surface. ${ }^{5}$ As such, the assumption that the metal-O bond is mostly covalent-argument on which the application of the $d$-band model relies-does not hold in general. The latter is also reproduced and substantiated by our Bader ${ }^{37}$ analyses of the DFT electronic valence charge density. As shown in Table II, there is an unbalanced charge transfer from metal atoms to $\mathrm{O}$ for the nine metal species that we have analyzed.

\section{B. Strength of the Au-O and Ag-O bonds: Force-constant and charge-density analyses}

The first problem we tackle is that of showing that the $d$ band model indeed gauges only $E_{d-h y b}$, and it does it correctly at least for Au and Ag. Namely, according to the $d$-band model, at least the covalent $\mathrm{Au}-\mathrm{O}$ bond must be stronger than the $\mathrm{Ag}-\mathrm{O}$ one: $\left|E_{d-h y b}(\mathrm{Au})\right|>\left|E_{d-h y b}(\mathrm{Ag})\right|$. The objective is, then, to probe the magnitude of $E_{d-h y b}$ alone. For this purpose, we turn here to the force constants of the metal-O bond. Force constants are in general indicative of bond strength. The adsorbatemetal force constants will be quite useful in the present undertake ${ }^{39}$ because they get the largest contribution from the covalent bond, whereas the ionic bond is known to influence them considerably less. ${ }^{40,41}$ The calculated force constants and corresponding vibrational frequencies are displayed in Table III. Briefly, in-plane force constants of the Au-O bond are 40\% larger than those of the $\mathrm{Ag}-\mathrm{O}$ bond. To corroborate these findings, we have as well analyzed the metal-O bonding charge for $\mathrm{Au}$ and $\mathrm{Ag}$. The covalent charge bridge along the $\mathrm{O}-\mathrm{Ag}$ and $\mathrm{O}-\mathrm{Au}$ bonds (see Fig. 2) clearly exhibits that there is a significant hybridization between the states of $\mathrm{O}$ and those of the these two metals. Of course, the charge density does not tell us about particular contributions of $s, p$, and $d$-electrons to the covalent charge bridge in Fig. 2. However, there are several facts suggesting that (1) the $d$-states do contribute to the covalent bond; namely, as mentioned earlier, owing to the $s$ - $d$ hybridization, there are unoccupied $d$-states in bulk Au. Moreover, the radial distribution of Au states is such that the $5 d$-density tail is high enough to overlap with that of $\mathrm{O}$, and yet the overlap is not too large so as to cause a large Pauli repulsion (see Ref. 42); (2) the Au 6s- and 6p-electrons contribute to the ionic $\mathrm{Au}-\mathrm{O}$ bonding rather than to the covalent $\mathrm{Au}-\mathrm{O}$ bond because, although relativistic, the $6 s$ electrons are quite delocalized. In fact, the largest $6 s$ density peak is wide enough to cover the entire adsorbed oxygen atom (see Ref. 42). The $6 p$-states should then be even more delocalized due to smaller relativistic effects. Furthermore, oxygen's electronegativity is much higher than that of Au. (The Pauling electronegativity of $\mathrm{O}$ is 3.44 while that of $\mathrm{Au}$ is 2.54).

Therefore, because the charge-density bridge in Fig. 2 accounts primarily for the hybridization between metal $d$ states and $\mathrm{O} p$-states, it is clear that attaching the nobility of gold to the mere Pauli-repulsion orthogonalization terms in the $d$-band model is not correct. As expected, the valence charge density along the bonds also shows that the Au-O covalent bond is stronger than the $\mathrm{Ag}-\mathrm{O}$ covalent bond 
TABLE II. Adsorption energy of oxygen $\left(E_{B}(O)\right)$ on (111) metal surfaces (values in parenthesis are for slabs of a $(3 \times 3)$ in-plane periodicity); Occupied $\left(\epsilon_{d}\right)$ and unoccupied $\left(\epsilon_{d^{*}}\right) d$-band center; Bader charges of oxygen $\left(q_{O}\right)$ and each of its metal neighbors $\left(q_{M}\right)$; the O-metal $\left(a_{0}\right)$ and metal-metal $\left(d_{M-M}\right)$ bond lengths (for the metal atoms binding with $\mathrm{O}$ only); the metals' work function $(\Phi)$; the characteristic radius of the metals' $d$-states $\left(r_{d}\right)$; fractional filling of the metals' $d$-band $(f)$.

\begin{tabular}{llccccccccc}
\hline \hline & $E_{B}(O)$ & $\epsilon_{d}(\mathrm{eV})$ & $\epsilon_{d^{*}}(\mathrm{eV})$ & $q_{O}(\mathrm{e})$ & $q_{M}(\mathrm{e})$ & $a_{0}(\AA)$ & $d_{M-M}(\AA)$ & $\Phi(\mathrm{eV})$ & $r_{d}(\AA)$ & $f$ \\
\hline $\mathrm{Cu}$ & $4.95(4.93)$ & -2.47 & 1.61 & 0.99 & 0.29 & 1.89 & 2.49 & 4.98 & 0.67 & 0.97 \\
$\mathrm{Ag}$ & $3.78(3.78)$ & -4.06 & 1.78 & 0.89 & 0.23 & 2.14 & 2.81 & 4.80 & 0.89 & 0.98 \\
$\mathrm{Au}$ & $3.37(3.40)$ & -3.37 & 1.69 & 0.78 & 0.19 & 2.13 & 2.78 & 5.31 & 1.01 & 0.97 \\
$\mathrm{Pd}$ & $4.63(4.62)$ & -1.82 & 1.26 & 0.70 & 0.22 & 2.01 & 2.73 & 5.64 & 0.94 & 0.90 \\
$\mathrm{Ni}$ & $5.70(5.71)$ & -1.71 & 1.04 & 0.87 & 0.26 & 1.84 & 2.44 & 5.50 & 0.71 & 0.87 \\
$\mathrm{Pt}$ & $4.51(4.52)$ & -2.43 & 1.08 & 0.77 & 0.23 & 2.04 & 2.71 & 6.10 & 1.04 & 0.88 \\
$\mathrm{Ru}$ & 6.28 & -2.48 & 0.98 & 0.87 & 0.23 & 2.02 & 2.57 & 5.58 & 1.05 & 0.71 \\
$\mathrm{Ir}$ & 5.09 & -2.91 & 0.70 & 0.93 & 0.28 & 2.06 & 2.67 & 5.76 & 1.08 & 0.80 \\
$\mathrm{Rh}$ & 5.33 & -2.27 & 0.82 & 0.77 & 0.22 & 2.01 & 2.66 & 5.32 & 0.99 & 0.80 \\
\hline \hline
\end{tabular}

(Fig. 2). In summary, the above results attest quantitatively that the $d$-band model holds but only when explicitly appraising the covalency of the $\mathrm{Au}-\mathrm{O}$ and $\mathrm{Ag}-\mathrm{O}$ bonds.

At this point, the question as to why $E_{B}(O)$ is smaller for $\mathrm{Au}$ than for $\mathrm{Ag}$ obviously directs the attention to the charge transferred from the metal to $\mathrm{O}$. We shall show later that the $\mathrm{Au}-\mathrm{O}$ bond is indeed less ionic than the $\mathrm{Ag}-\mathrm{O}$ one, as one could expect from the surfaces' work function or even from atomic ionization energies (both consequences of the relativistic effects). Thus, it will be imperative to determine whether the $d$-band model fails to account for the differences in $E_{B}(O)$ between $\mathrm{Au}$ and $\mathrm{Ag}$ only because of such differences in ionicity. We shall demonstrate that the high work function of $\mathrm{Au}$ and the consequent low ionicity of the bond are not sufficient to explain the low $E_{B}(O)$ on $A u$. But before doing that, we will now provide evidence that $A u$ is less reactive than Ag because of the large electronic and thus structural perturbation produced by adsorbates on its surfaces.

\section{The perturbation energy from ab initio total-energy calculations}

The binding energy of an adsorbate is determined by the reduction of the system's total energy upon formation of the chemical bond between the adsorbate and its neighboring substrate atoms. Bond formation certainly reduces the total energy. Nonetheless, as an adsorbate binds to a surface, it causes a redistribution of the valence charge density from the surface and subsurface regions toward the adsorbate in order to form a covalent charge bridge or/and ionic charge transfer between the adsorbate and those neighboring metal surface atoms. Such charge density perturbation thus affects the bonding charge

TABLE III. Symmetrized force constants $k_{x x}, k_{y y}$, and $k_{z z}$ of oxygen on $\mathrm{Au}(111)$ and $\mathrm{Ag}(111)$ given in $\mathrm{eV} / \AA^{2} . k_{x x}$ and $k_{y y}$ are the in-plane force constants. Vibrational frequencies in meV of the parallel $\left(\omega_{\|}\right)$and vertical $\left(\omega_{\perp}\right)$ metal-O vibrational modes.

\begin{tabular}{cccccc}
\hline \hline & $k_{x x}$ & $k_{y y}$ & $k_{z z}$ & $\omega_{\|}$ & $\omega_{\perp}$ \\
\hline$A u-O$ & -7.31 & -7.24 & -8.15 & 43.5 and 43.7 & 46.1 \\
$A g-O$ & -5.18 & -5.35 & -7.85 & 36.4 and 36.8 & 45.3 \\
\hline \hline
\end{tabular}

among surface and subsurface atoms, which increases the energy of the system and effectively reduces the binding energy of the adsorbate. In turn, the surface responds to this charge redistribution through lattice distortions or reconstruction. Surface reconstruction/relaxation is a subject extensively studied for many substrates and adsorbates. In fact, many studies emphasize that not all substrates respond in the same manner to a given adsorbate. However, little attention has been paid to the fact that such varied responses from the substrate may prevent us from grasping the local bond strength of an adsorbate (either covalent or ionic) via the measured heat of adsorption or the calculated binding energy. So far, some works have reported the so-called relaxation energy $\left(E_{r x}\right)$ of an adsorbed surface, which is the energy contribution to the calculated $E_{B}(O)$ obtained by allowing to relax the interatomic forces on substrate atoms of the composite system. . $^{17,30,35,43,44}$ One way to calculate $E_{r x}$ involves the total energy of (1) the totally relaxed O-metal system $\left(E_{\text {total }}^{R}(O /\right.$ metal $\left.)\right)$ and (2) the O-metal system in which the substrate atoms are kept in the same positions as in the clean substrate $E_{\text {total }}^{U}(O /$ metal $)$ : $E_{r x}=E_{\text {total }}^{R}(O /$ metal $)-E_{\text {total }}^{U}(O /$ metal $) .{ }^{17,35,44}$ This energy has been found important for O-adsorption on 100-atom transitionmetal clusters, particularly for Au and Pt ones. ${ }^{44}$ In our calculations, inspection of the structural distortions undergone by the metal substrates upon O-adsorption clearly hints that the $\mathrm{O}$-induced disturbance is significantly stronger for Au than for any other metal. And yet, the distortion of the lattice caused by an adsorbate is a feature often neglected when rationalizing the binding energy of adsorbates within the $d$-band model. $E_{r x}$ was originally neglected because it is relatively small

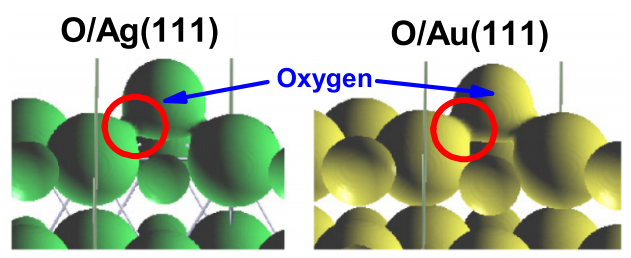

FIG. 2. Calculated three-dimensional charge-density isosurfaces of $\mathrm{O} / \mathrm{Ag}(111)$ (left) and $\mathrm{O} / \mathrm{Au}(111)$ (right) obtained for the same isovalue $\left(0.46 e / \AA^{3}\right)$ showing that the amount of valence charge bridging the $\mathrm{Au}-\mathrm{O}$ bond is larger than that bridging the $\mathrm{Ag}-\mathrm{O}$ bond (This is true regardless of the chosen isovalue). In both cases, $\mathrm{O}$ sits at the hollow site. 
TABLE IV. Relaxation energy $\left(E_{r x}\right)$ of the metal surfaces using a slab of a $(2 \times 2)$ in-plane periodicity. Values obtained for slabs of a $(3 \times 3)$ in-plane periodicity are given in parenthesis.

\begin{tabular}{cccccc}
\hline \hline & $E_{r x}(\mathrm{meV})$ & & $E_{r x}(\mathrm{meV})$ & & $E_{r x}(\mathrm{meV})$ \\
\hline $\mathrm{Cu}$ & $-120(-138)$ & $\mathrm{Pd}$ & -75 & $\mathrm{Ru}$ & -180 \\
$\mathrm{Ag}$ & $-100(-117)$ & $\mathrm{Ni}$ & -73 & $\mathrm{Ir}$ & -133 \\
$\mathrm{Au}$ & $-250(-270)$ & $\mathrm{Pt}$ & $-170(-189)$ & $\mathrm{Rh}$ & -105 \\
\hline \hline
\end{tabular}

for CO adsorption: ${ }^{17}$ The authors found that, for adsorbed $\mathrm{CO}, E_{r x}$ is smaller than $50 \mathrm{meV}$, as one would expect for weak chemisorption. Yet, this is certainly not the case for $\mathrm{O}$ adsorption, as shown in Table IV. But more importantly, a critical and clear distinction ought to be made between the relatively small ion-force-relaxation energy $E_{r x}$ and $E_{p e r t}$, which is defined as the overall drop in binding-energy associated with the perturbation to both the substrate geometry and electronic charge density, as mentioned in the beginning of the section. In other words, the lattice distortions seen in chemisorbed systems derive in the first place from the charge rearrangements occurring within the metal upon formation of the metal-O bond.

The difficulty in determining $E_{\text {pert }}$ lies in that the overall perturbation energy can neither be measured nor obtained via ground-state calculations because it involves non-equilibrium electronic processes. Hence, we need to devise a method for calculating $E_{\text {pert }}$. The main challenge behind assessing $E_{\text {pert }}$ is that of obtaining the charge density of the perturbed metal substrate $\rho_{P}($ metal $)$. This task demands to remove from the substrate the charge taken by oxygen upon adsorption to make the ionic and the covalent bond. In other words, one would wish to remove the charge "belonging" to the adsorbed oxygen from the charge density of the O-metal composite system, $\rho_{R}$ (adsorbate/metal) (Of course, the charge density "belonging" to an adsorbed atom cannot be unambiguously defined in principle). So far, however, our available tools only allow us to subtract the charge density of atomic oxygen, $\rho_{I}\left(\right.$ adsorbate), from $\rho_{R}($ adsorbate/metal) (Though we are planning to work in an algorithm to sort out the charge in the Wigner-Seitz sphere around oxygen or that given by Bader analyses). In analyzing whether $\rho_{I}(O)$ must be spin polarized or not, we consider the following: Our spinpolarized calculations indicate that, although atomic $\mathrm{O}$ is spin-polarized, none of the atoms of the composite system is spin polarized. Because this information derives from projecting the plane wave functions of the composite system into individual atomic orbitals, we conclude that (1) the overall charge density associated with oxygen that we need to remove from the composite system must be non-spin polarized and (2) the charge density of the perturbed substrate we are searching $\left(\rho_{P}(\right.$ metal $\left.)\right)$ must be non-spin polarized as well. Based on the above considerations, $E_{\text {pert }}$ is obtained $a b$ initio by performing the following steps: (1) We relax the chemisorbed system to obtain its charge density distribution, $\rho_{R}$ (adsorbate/metal). In this situation, the metal surface is subjected to the structural distortion and the perturbation to the electronic charge density brought in by the adsorbate $(\mathrm{O}$ in the present case). (2) We obtain the charge density of the isolated adsorbate species (located at the same position as in step (1) and non-spin polarized if all atoms are nonmagnetic in the composite system), $\rho_{I}($ adsorbate). (3) We then obtain the perturbed electronic charge density as $\rho_{P}$ (metal) $=\rho_{R}($ adsorbate $/$ metal $)-\rho_{I}($ adsorbate $)$. (4) Then, we calculate the total energy of the perturbed metal surface (without the adsorbate) by using and keeping fixed both the ionic distortion obtained in step (1) and the perturbed electronic charge density $\rho_{P}($ metal $)$ obtained in step (3). We denote this energy as $E_{\text {total }}^{P}($ metal). (5) In parallel, we obtain the total energy of the clean and thoroughly relaxed surface, $E_{\text {total }}^{R}$ (metal). (6) Finally, $E_{\text {pert }}$ is obtained as the difference in total energy between the relaxed (step (5)) and the perturbed surface (step (4)).

$$
E_{\text {pert }}=E_{\text {total }}^{R}(\text { metal })-E_{\text {total }}^{P}(\text { metal }) \text {. }
$$

Remarkably, the above calculations yield $E_{\text {pert }}$ to be $1.52 \mathrm{eV}$ for $\mathrm{Au}(111)$. This is to be contrasted with the value of the mere $E_{r x}$ which, although significant, is only $0.25 \mathrm{eV}$. This shows that the largest contribution to $E_{\text {pert }}$ comes from the electron-density perturbation. More importantly, the ab initio calculated $E_{\text {pert }}$ for $\mathrm{Ag}, 0.97 \mathrm{eV}$, is significantly smaller than for Au. Naturally, obtaining $E_{\text {total }}^{P}$ by subtracting the charge density of atomic $\mathrm{O}$ inherently involves the work functionwhich is relevant for the ionic bond-and not only the charge rearrangement within the volume occupied by the metal atoms of the chemisorbed surface. This introduces a doublecounting error that, moreover, complicates the comparison among results for highly ionic and highly covalent bonds. Nevertheless, comparisons among $E_{\text {pert }}$ for surfaces of species that transfer the same amount of charge to $\mathrm{O}$, indicate that $E_{p e r t}$ is proportional to $E_{r x}$. For instance, the calculated $E_{p e r t}$ for both $\mathrm{Au}$ and $\mathrm{Pt}$ is six times larger than their corresponding $E_{r x}$. We shall use this result later on to find the magnitude of the various factors influencing $E_{B}(O)$.

\section{Insights into the perturbation energy from metal-0 molecules}

We now turn to corroborate the large magnitude of $E_{\text {pert }}$ for $\mathrm{Au}$. Let us consider the O-metal binding strength in a system that (1) allows us to calculate $E_{d-h y b}+E_{\text {ionic }}$ from first principles and, at the same time, (2) does not let the perturbation to the $\mathrm{Au}-\mathrm{Au}$ bonds intervene: the metal-O molecule. Surprisingly, we find that, although the $\mathrm{O}-\mathrm{Ag}(111)$ bond is stronger than the $\mathrm{O}-\mathrm{Au}(111)$ bond by $0.41 \mathrm{eV}$, the reverse trend holds for the corresponding metal-O molecules. The $\mathrm{O}-\mathrm{Ag}$ molecule binding energy $(2.55 \mathrm{eV})$ is smaller than the $\mathrm{O}-\mathrm{Au}$ one $(2.92 \mathrm{eV})$ by $0.37 \mathrm{eV}$. This means that there is a binding-energy turnover of $\sim 0.8 \mathrm{eV}$. In other words, $E_{\text {pert }}$ for $\mathrm{Au}(111)$ should then be at least $\sim 0.8 \mathrm{eV}$, which is in agreement with our above $a b$ initio calculations of $E_{\text {pert }}$. The latter is remarkable also because the $\mathrm{Ag}-\mathrm{O}$ bond is even more ionic than the $\mathrm{Au}-\mathrm{O}$ bond in the molecule than in the (111) surface. In the molecule Ag transfers to O $25 \%$ more charge than $\mathrm{Au}$, while on the surface this difference is reduced to 13\% (see Table II). Thus, these molecules-which inherently isolate the perturbation energy from the energy of the ionic and covalent bonding - not only substantiate the magnitude of $E_{\text {pert }}$ but also reveal that the adsorbate-induced perturbation 
is the key to understand Au inertness-and not the relatively low ionicity of the $\mathrm{Au}-\mathrm{O}$ bond.

\section{E. The large electronic perturbation on $\mathrm{Au}(111)$ upon $O$ adsorption: $A$ charge density analysis}

Our ab initio simulations of the charge-density relaxation induced by the mere presence of $\mathrm{O}$ also confirm that the electronic perturbation taking place around the surface upon $\mathrm{O}$ adsorption is larger for Au than for Ag (see Fig. 3). Notice then that the charge relaxation induced by the pure structural distortion (that associated with $E_{r x}$ ) is neglected in these plots. Fig. 3 displays selected planes sampling the difference $\Delta \rho$ between the charge density of the $\mathrm{O}$-adsorbed metal surface and that of a clean metal surface that is kept in the distorted geometry induced by O. Fig. 3 shows that upon the formation of the covalent and ionic metal-O bonds (1) charge depletion of the metal-metal bonds is the dominant effect $^{45}$ on the substrate and (2) such charge depletion is significantly larger for Au than for Ag. In particular, Figs. 3(a) and 3(b) demonstrate that Au undergoes a quite large charge depletion of bonding charge among surface atoms, whereas the depletion is practically inexistent at the corresponding region of $\operatorname{Ag}(111)$. Notice that the surface intralayer charge depletion is the largest for $\mathrm{Au}$. The latter finding is in line with two facts (1) the largest structural distortion sustained by $\mathrm{Au}(111)$ is that of the bonds at the surface layer and (2) the largest contribution to $E_{r x}$ derives precisely from that distortion. Of course, the depletion of $\mathrm{Au}-\mathrm{Au}$ bonding charge density directed toward the adsorbed $\mathrm{O}$ is not so evident because the $\mathrm{Au}-\mathrm{O}$ hybridization occurs at the same location. In the case of $\mathrm{Ag}(111)$, the largest charge depletion occurs between the

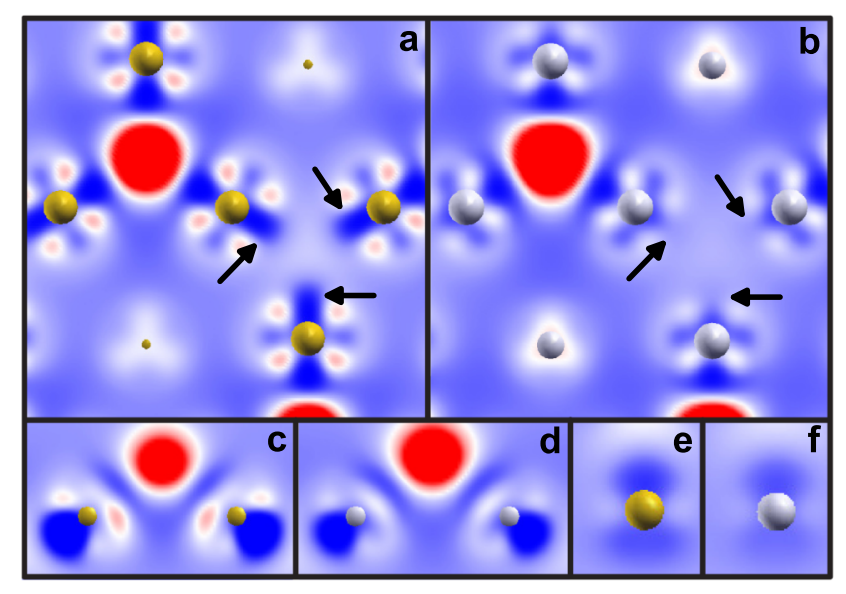

FIG. 3. Contour plots of the calculated valence charge-density redistribution, $\Delta \rho$, upon removal of adsorbed $\mathrm{O}$ while keeping the lattice distortion induced by O: Comparison between $\mathrm{Au}(111)$ and $\mathrm{Ag}(111)$. The yellow and grey balls represent $\mathrm{Au}$ and $\mathrm{Ag}$ atoms, respectively. The blue and red tones represent charge depletion and accumulation, respectively, upon $\mathrm{O}$ adsorption. $\mathrm{O}$ atoms are not displayed but, in plots (a)-(d), the big red spots indicating a large charge accumulation signalize its location. White signifies that no change in the charge takes place. (a) ( $\mathrm{Au})$ and (b) (Ag) display $\Delta \rho$ at a plane that is parallel to the surface at almost the same height as the surface atoms. The arrows indicate the regions to be compared between (a) (Au) and (b) (Ag). (c) (Au) and (d) (Ag) display $\Delta \rho$ at a plane that is perpendicular to the surface around the surface atoms bound to $\mathrm{O}$. (e) (Au) and (f) (Ag) display $\Delta \rho$ at the same plane as before but around the sub-surface atoms. The scale in all plots varies from -0.06 to $0.06 e \AA^{3}$. surface and subsurface layers. Yet, the corresponding one for Au is larger as well, as shown by Figs. 3(c) and 3(d). In fact, that the depletion of bonding charge upon $\mathrm{O}$ adsorption is larger for $\mathrm{Au}$ than for $\mathrm{Ag}$ is noticeable even around subsurface atoms, as shown by Figs. 3(e) and 3(f). Finally, it is important to emphasize that the charge-density redistribution around the metal-metal bonds, which is primarily depletion, accounts for the difference between $E_{p e r t}$ and $E_{r x}$ (since $E_{r x}$ derives only from the structural distortion). The latter speaks in favor of our earlier results: $E_{\text {pert }}$ is larger for Au than for Ag mainly because of a stronger electronic perturbation.

\section{F. Identifying the contributions of $E_{\text {ionic }}, E_{d-h y b}$, and $E_{\text {pert }}$ to the $a b$ initio $E_{B}(0)$ : A back analysis}

To reaffirm quantitatively that the large perturbation inflicted on $\mathrm{Au}$ is responsible for its oxidation resistance, we have also made a back analysis of the ab-initio $E_{B}(\mathrm{O})$ using a multiple regression model from data on eight input variables affecting the metal-O bond. These variables are related either to the covalent bond, the ionic one, or the perturbation on the metal bonds. The back analysis allows us to express the $a b$ initio $\mathrm{O}$ binding energy as $E_{B}(\mathrm{O})=E_{d-h y b}+E_{\text {ionic }}+E_{\text {pert }}$ and identify by how much each of these components affect $E_{B}(O)$ for nine metal surfaces. In the case of the perturbation to the metal bonds, we shall consider that the (negative) contribution to $E_{B}(O)$ from $E_{\text {pert }}$ is indeed proportional to $E_{r x}$ for all species, $E_{p e r t}=\chi E_{r x}$, as suggested by our $a b$ initio calculations of $E_{p e r t}$. Thus, $E_{r x}$ is treated as one of the input variables and the proportionality coefficient $\chi$ is to be determined (see the Appendix). The other two contributions to $E_{B}(O), E_{d-h y b}$ and $E_{i o n i c}$ are modeled analytically in terms of other seven input parameters that are mostly obtained from ab initio calculations (see the Appendix). Table II displays all input parameters used in the multiple regression and the results of this are summarized in Fig. 4. We remind the reader that the procedure of calculating $E_{\text {pert }}$ and $E_{\text {ionic }}$ involves a double counting incident because, in calculating the ab initio $E_{\text {pert }}$, subtracting the charge density of atomic $\mathrm{O}$ inherently leaves in a vacuum region the charge transferred to $\mathrm{O}$, whose energy cost is already accounted for in the work function, which in turn is also considered in $E_{\text {ionic }}$. However, we shall see that the error in the $a b$ initio $E_{\text {pert }}$ can be estimated from the present back analysis because it is totally independent from the $a b$ initio $E_{\text {pert }}$.

Fig. 4 displays by how much the covalent and the ionic contributions increase $E_{B}(O)$ and by how much $E_{\text {pert }}$ decreases it for nine transition metals. As such, there are several features implied in it that are important to highlight: (1) First of all, $\mathrm{Au}$ displays the largest $E_{\text {pert }}$ among the nine metals considered here. It amounts $\sim 1 \mathrm{eV}$, which is in agreement with our earlier estimations and with the fact that the distortion is significantly stronger for Au than for any other metal. (2) Contrary to the expected "nobleness" of gold, the covalent-bond energy curve shows that, from all three group-11 metals, $\mathrm{Au}$ is in fact the least noble inasmuch as it displays the strongest covalent bond with $\mathrm{O}$. The most striking aspect of this is that the $\mathrm{Au}-\mathrm{O}$ covalent bond is stronger than the $\mathrm{Cu}-\mathrm{O}$ one. Therefore, in this case, the $d$-band center fails again because the $d$-band 


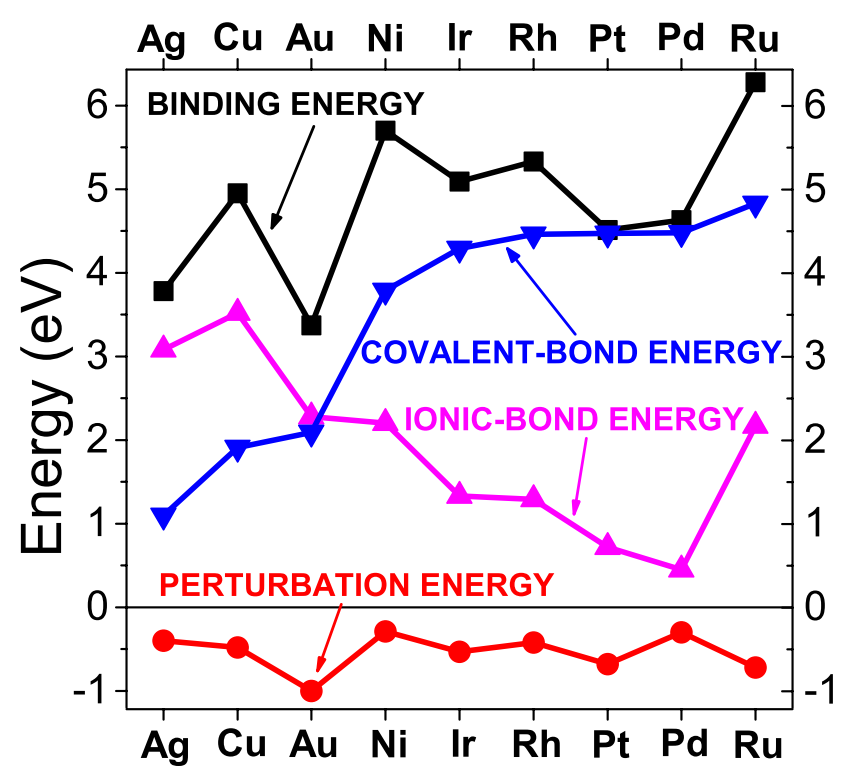

FIG. 4. Binding energy of oxygen $\left(E_{B}(O)\right)$ on nine (111) metal surface and the contributions to it from the covalent bond $\left(E_{d-h y b}\right)$, the ionic bond $\left(E_{\text {ionic }}\right)$, and the perturbation on the metal bonds $\left(E_{\text {pert }}\right)$. Elements are ordered according to the covalent-bond energy, which increases from left to right.

center is closer to $\mathrm{E}_{F}$ for $\mathrm{Cu}$ than for $\mathrm{Au}$ (Table II). The reason is that the interatomic matrix element for the $\mathrm{Au}-\mathrm{O}$ bond is larger than that for the $\mathrm{Cu}-\mathrm{O}$ one. The latter in turn is due to the quite long orbitals of Au (i.e., large $r_{d}$ ). Specifically, the large $r_{d}$ of Au does weaken the bond because of the orthogonalization-energy cost, ${ }^{16}$ but overall it enhances the hybridization between $\mathrm{Au}$ and $\mathrm{O}$ states. Notice that the long $r_{d}$ of $\mathrm{Au}$ is due in part to the relativistic effects. Thus, in this sense, the relativistic effect on the $d$-orbitals makes Au not more noble but less noble than $\mathrm{Cu}$. (3) As expected from the differences in work function, the O-binding energy on Ag gets a much larger contribution from the ionic bond than Au does. Nonetheless, even if we consider the covalent plus the ionic contributions, $E_{d-h y b}+E_{\text {ionic }}$, one finds that the $\mathrm{Ag}-\mathrm{O}$ bond is still weaker than the $\mathrm{Au}-\mathrm{O}$ bond by $\sim 0.2 \mathrm{eV}$. Therefore, the contribution to $E_{B}(O)$ from $E_{\text {ionic }}$ (i.e., the relativistic effects on the $s$ and $p$-orbitals) is also unable to resolve the controversial differences in reactivity between $\mathrm{Au}$ and $\mathrm{Ag}$ or project a rationale for the nobleness of gold. (4) $E_{d-h y b}+E_{\text {ionic }}$ (or $E_{B}(O)-E_{\text {pert }}$ ) would imply a relatively large $E_{B}(O)$ of $4.4 \mathrm{eV}$ for $\mathrm{Au}(111)$, even though the $\mathrm{Au}-\mathrm{O}$ ionic bond is quite weak with respect to the $\mathrm{Ag}-$ and $\mathrm{Cu}-\mathrm{O}$ ones and the $\mathrm{Au}-\mathrm{O}$ covalent bond is also quite weak with respect to those between $\mathrm{O}$ and all other transition metals. Therefore, if not for the large perturbation energy, $\mathrm{Au}-\mathrm{Au}$ and $\mathrm{O}_{2}$ bonds would be much more unstable with respect to $\mathrm{Au}-\mathrm{O}$ bonds and, thus, gold would dissociate $\mathrm{O}_{2}$ and oxidize more readily than it actually does, as in the case of $\mathrm{Cu}$ and $\mathrm{Ag}$, both for which $E_{B}(O)$ is much larger than their corresponding cohesive energy and than $\frac{1}{2} E\left(O_{2}\right)$. (5) The strength of the metal-O with respect to the metal-metal bond may alone be decisive for corrosion. For example, $E_{B}(O)$ on $\mathrm{Cu}$ is weaker than that on $\mathrm{Rh}$; yet $\mathrm{Rh}$ is more noble than $\mathrm{Cu}$ because the $\mathrm{Rh}-\mathrm{O}$ bond is weaker than the $\mathrm{Rh}-\mathrm{Rh}$ bond (the opposite is true for $\mathrm{Cu}$ ). (6) $\mathrm{Cu}$ makes the most ionic bond with $\mathrm{O}$ among the nine transition metals. The remarkable strength of the ionic bond between
$\mathrm{O}$ and $\mathrm{Cu}$ is in fact the main reason for which $E_{B}(O)$ on $\mathrm{Cu}$ exceeds not only that on Au but also those on Pt and Pd (Of course, it is also important that the $\mathrm{Cu}$ surface does not suffer a large perturbation). Thus, the $d$-band model is in general inappropriate to describe the reactivity differences between $\mathrm{Cu}$ and $\mathrm{Pd}$ or $\mathrm{Pt}$ because the $\mathrm{Cu}-\mathrm{O}$ is definitely not predominantly covalent. (7) $E_{B}(O)$ for Pd is relatively small despite of the expected and actual strong covalent bond. The reason is that Pd bears the weakest ionic bond with O. So, even though $E_{\text {pert }}$ reaches its minimum for $\mathrm{Pd}$ (and $\mathrm{Ni}$ ), $0.3 \mathrm{eV}, E_{\text {ionic }}$ is hardly enough to compensate it. Pd is perhaps the only case for which indeed the binding energy is predominantly covalent. (8) As expected, $E_{d-h y b}$ is dominant for group-8, -9 , and - 10 elements but not for group-11 metals, including $\mathrm{Au}$ (whose bond with $\mathrm{O}$ is practically as much ionic as covalent). Although the covalent-bond energy is fairly constant for group-9 and -10 elements, the ionic-bond energy decreases to its minimum values for group-10 elements ( $\mathrm{Pd}$ and $\mathrm{Pt}$ ). (9) The $E_{\text {ionic }}$ is dominant for $\mathrm{Ag}$ and $\mathrm{Cu}$. $E_{\text {ionic }}$ drops significantly for $\mathrm{Au}$ and $\mathrm{Ni}$, and even more for group- 9 elements; yet, it reaches its minimum for Pt and Pd (group-10 elements as Ni). However, $E_{\text {ionic }}$ increases again for $\mathrm{Ru}$ (group-8 element). In fact, the $\mathrm{Ru}-\mathrm{O}$ bond is as ionic as the $\mathrm{Ni}-\mathrm{O}$ one.

Note that the ab initio $E_{\text {pert }}$ is $\sim 0.5 \mathrm{eV}$ larger than the value estimated from the back analysis of $E_{B}(O)$ and from the analysis of the O-metal dimer. We attach this discrepancy to the energy cost (work function) accounted in the ab initio $E_{\text {pert }}$ for the charge transferred into the vacuum. Thus, this discrepancy of $\sim 0.5 \mathrm{eV}$ is an estimate of the double-counting error that would result from adding the ab initio $E_{\text {pert }}$ plus $E_{\text {ionic }}$ Nevertheless, it is worth to highlight that we are not limited by such double-counting error because we have estimated the magnitude of $E_{\text {pert }}$ through other two totally independent procedures: the O-metal dimers and the numerical fitting (in which only $E_{r x}$ enters as a descriptor). Importantly, all three methods yield $E_{\text {pert }}$ of the order of $1 \mathrm{eV}$ for $\mathrm{O} / \mathrm{Au}(111)$.

\section{G. Analysis of the electronic density of states (DOS) and its relation to $E_{p e r t}$}

Although the $d$-band center alone cannot characterize the O-metal bond strength, it is beyond question that the spatial and energetic distribution of electronic states (the electronic structure) underlies the trends in all three parts contributing to the binding energy of adsorbates $\left(E_{\text {ionic }}, E_{d-h y b}\right.$, and $\left.E_{\text {pert }}\right)$, as well as in many other properties of materials. Thus, in this section, we report the correlation that exists between the magnitude of $E_{\text {pert }}$ and some of the particulars of the density of states of the nine metals we have addressed. Then, we analyze in detail the redistribution of the density of $d$-states of surface $\mathrm{Au}$ and $\mathrm{Ag}$ atoms upon O-adsorption, as a first step to explain why $\mathrm{Au}$ displays the largest $E_{\text {pert }}$ among the nine transition metals that we have investigated.

We first note that Au has a relatively deep $d$-band that slightly extends to energies below $-6 \mathrm{eV}$ and has a marginal amount of $d$-states around $E_{F}$. The perturbation is milder$E_{\text {pert }} \sim 0.7 \mathrm{eV}$-for those metals that have likewise some deep $d$-states but also a large amount of states at $E_{F}, \mathrm{Pt}$ and Ru. It is even milder for those metals whose $d$-band is narrower and 
confined above $-6 \mathrm{eV}$ and has a marginal amount of $d$-states around $E_{F}, \mathrm{Cu}$ and $\mathrm{Ag}$. Finally, the least affected, $\mathrm{Pd}$ and $\mathrm{Ni}$, have most of their bonding states above $-5 \mathrm{eV}$ and, at the same time, have high DOS at $E_{F}$.

The above trends suggest that the magnitude of $E_{\text {pert }}$ can be traced to the position of the bonding and antibonding $d$-states because that determines different degrees of energy overlapping between the states of $\mathrm{O}$ and those of the metal; namely, hybridization of the O-states with the deepest metal-states depletes mainly the metal bonding states and thus weakens the metal-metal bonds, whereas hybridization with the states closest to $E_{F}$ would mostly consume metal antibonding states, thus stabilizing the metal-metal bond. Of course, to attest the above rationale a quantitative analysis of the bonding and antibonding contribution of particular orbitals would be in order. However, as a first step, one can analyze the changes in the electronic DOS taking place in a bulk atom as it passes to be a surface atom upon creation of a surface and then the changes in the DOS when that surface atom becomes bound to $\mathrm{O}$. Even this simple approach involves the analysis of a large amount of data. However, we shall concentrate only on the surface-atom $d$-states that turn to be parallel to the surface because (1) the most dramatic electronic perturbation happens along the surface intralayer $\mathrm{Au}-\mathrm{Au}$ bonds (Figs. 3(a) and 3(b)), and (2) such charge depletion also establishes the largest difference between $\mathrm{Au}$ and $\mathrm{Ag}$. Here, we analyze the projected- $d_{x^{2}-y^{2}}$ states (Fig. 5), which for some atoms in the supercell spread along the same direction as the charge depletion shown in Fig. 3(a). Fig. 5(a) shows that the energy distribution of bulk Au states significantly changes upon the formation of the (111) surface. Specifically, they lose a significant amount of bonding states (particularly below $-5 \mathrm{eV}$ with respect to $E_{F}$ ) and gain anti-bonding states (particularly above $-2 \mathrm{eV}$ with respect to $E_{F}$ ). The loss of bonding states is mostly compensated by the population of states at $-5.5,-4.80$, and $-3.75 \mathrm{eV}$ (still in the range of being bonding states). Then, upon O-adsorption, the lowest-energy states are re-populated (Fig. 5(b)). That re-population is clearly not related to the $\mathrm{Au}-\mathrm{Au}$ bonding, but to the relatively strong hybridization with the $p$ states of $\mathrm{O}$ (see Fig. 5(c)). More outstandingly, all the DOS peaks created to strengthen the intralayer bonds upon the creation of the surface (at $-3.75,-4.80$, and $-5.5 \mathrm{eV}$ ) are totally depleted by $\mathrm{O}$. As expected, something similar happens to Ag but to a much lesser degree (see Figs. 5(d)-5(f)). First of all, Ag does not acquire as many anti-bonding states upon the creation of the surface but also does not gain any significant amount of bonding states at intermediate energies (Fig. 5(d)). The re-population of low-energy Ag states (below $-4.5 \mathrm{eV}$ with respect to $E_{F}$ ) is relatively poor (Fig. 5(e)). This is because the hybridization with the $p$-states of $\mathrm{O}$ is also very poor (Fig. 5(f)), which is in agreement with our previous conclusions that the covalent $\mathrm{Au}-\mathrm{O}$ bond is stronger than the $\mathrm{Ag}-\mathrm{O}$ bond. But most importantly, $\mathrm{Ag}(111)$, unlike $\mathrm{Au}(111)$, does not undergo the large depletion of low- and intermediate-energy peaks as $\mathrm{Au}(111)$ does upon adsorption of $\mathrm{O}$. The latter is also in agreement with the fact that $E_{\text {pert }}$ and the charge depletion are both larger for $\mathrm{Au}$ than for $\mathrm{Ag}$.

The latter analysis supports that the particulars of the energy distribution of Au $d$-states ultimately determine its apparent nobleness. Specifically, $E_{\text {pert }}$ is particularly large for Au because of its wide and deep $d$-band. It is much wider than that of $\mathrm{Ag}$ and $\mathrm{Cu}$, which allows for a large energy splitting between the bonding and antibonding states of $p$-O. This feature favors the hybridization between $d$-Au bonding states and $p$-O states, which ultimately causes the large charge depletion suffered by Au surface atoms. Still, because the $d$-band of $\mathrm{Au}$ is deep and thus has a negligible amount of DOS around $E_{F}$ - just as those of $\mathrm{Ag}$ and $\mathrm{Cu}$, the antibonding states of $\mathrm{O}$ do not reach to hybridize much with the top of its $d$-band and thus do not deplete the Au antibonding states.

\section{DISCUSSION}

Gold's corrosion resistance is well known to everyone and experiments can certainly confirm the low heat of adsorption of highly electronegative adsorbates on its surfaces. However, the currently accepted rationales for its apparent unique nobleness are not satisfactory. We have shown that the inertness of gold is explained neither by the strength of the local adsorbate-metalatom covalent interaction nor by the relation between the surface $d$-band center and reactivity. The relativistic effect on the $6 s$ and $6 p$ electrons of Au yields a weak ionic bond with $\mathrm{O}$ but is not enough to explain the small heat of adsorption. In fact, this relativistic effect is compensated by that on the $5 d$ electrons. The latter is responsible for gold's ability to make stronger covalent bonds than $\mathrm{Ag}$ and $\mathrm{Cu}$. This is illustrated by gold's cohesive energy, the $\mathrm{Au}-\mathrm{H}$ molecule bond, ${ }^{2}$ and the $\mathrm{Au}-\mathrm{O}$ molecule bond.

Here, we advance that gold's apparent inertness, while in part aided by its almost totally filled $d$-band, is rooted in the large energy cost associated with the large perturbation undergone by the surface upon adsorption of highly electronegative species, and not necessarily because of a weak adsorbate-metal interaction. Specifically, first of all, contrary to the so far accepted understanding of Au nobleness and despite the singular small binding energy between $\mathrm{O}$ and $\mathrm{Au}$ surfaces, our charge-density and force-constant analyses show that the actual covalent $\mathrm{Au}-\mathrm{O}$ bond is stronger than the covalent $\mathrm{Ag}-\mathrm{O}$ and $\mathrm{Cu}-\mathrm{O}$ bonds. This finding actually concurs with the fact that in the bulk, $\mathrm{Au}-\mathrm{Au}$ bonds are stronger than $\mathrm{Cu}-\mathrm{Cu}$ and $\mathrm{Ag}-\mathrm{Ag}$ bonds, as seen from their corresponding cohesive energies. Then, although the covalent bond accounts for an important fraction of the metal-O binding energy and can be estimated via the $d$-band model, we demonstrate that the metal-O bond is not necessarily predominantly covalent but it is also partially ionic. Moreover, the contribution from the ionic metal-O bond to the overall binding is often quite significant. In fact, the contributions to the binding energy from the covalent bond plus that from the ionic one make $\mathrm{Au}-\mathrm{O}$ bonds, in principle, much more stable than the $\mathrm{Au}-\mathrm{Au}$ bonds. Interestingly though, because the $\mathrm{Au}-\mathrm{O}$ ionic bond is notoriously weaker than the $\mathrm{Ag}-\mathrm{O}$ one, one could think that the relatively low ionicity of the $\mathrm{Au}-\mathrm{O}$ bond is the root cause of $\mathrm{Au}$ inertness. This is not the case. $\mathrm{Au}-\mathrm{O}$ and $\mathrm{Ag}-\mathrm{O}$ molecules - which inherently isolate the perturbation energy from that of the ionic and covalent bonding-reveal that the crux of the matter to explain the O-binding-energy difference between $\mathrm{Au}(111)$ and $\operatorname{Ag}(111)$ is the geometric 

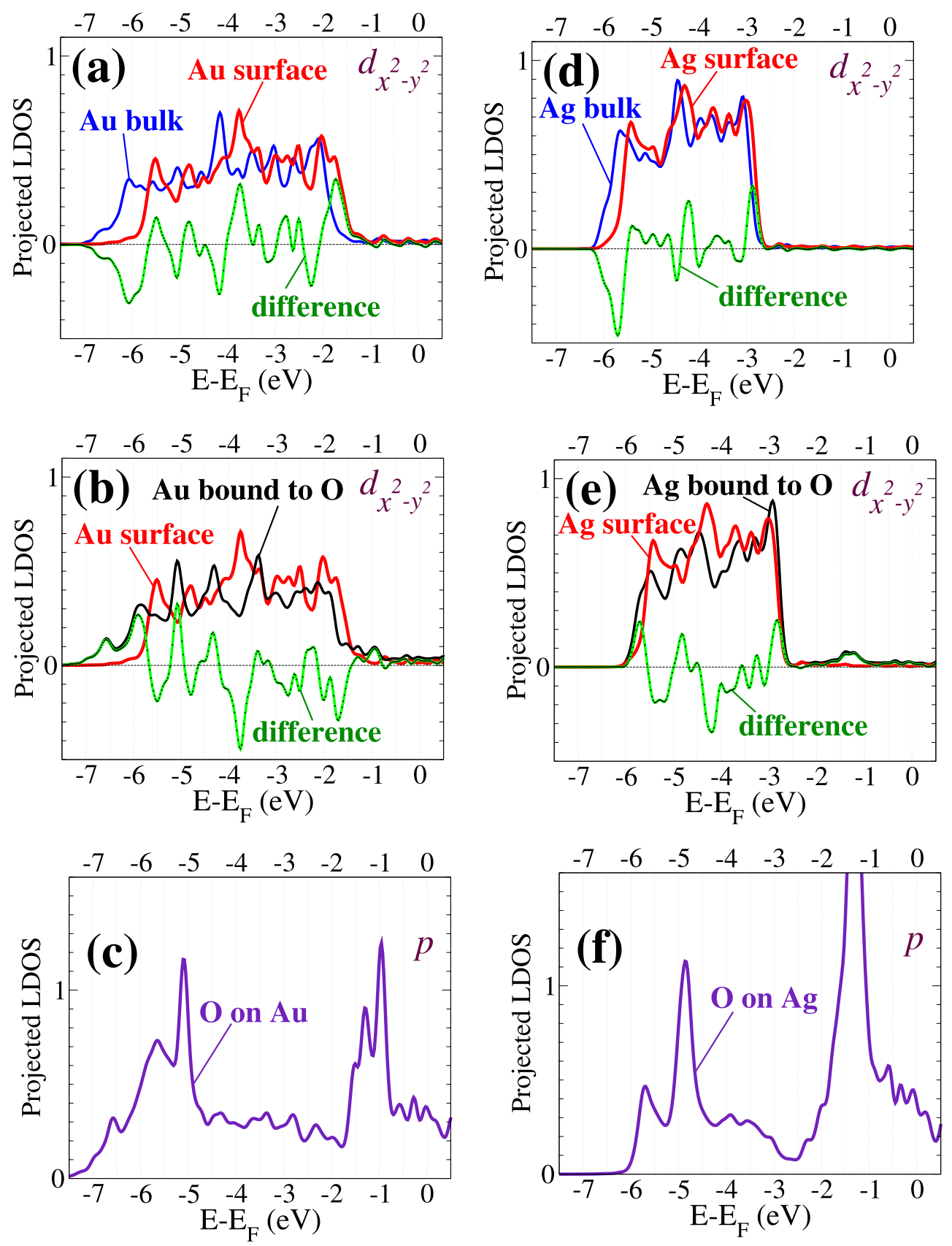

FIG. 5. Projected- $d_{x^{2}+y^{2}}$ electronic density of states (number of states per atom and per eV) of (a) bulk Au atom (blue), clean Au(111) atom (red), and the difference between them (green-black); (b) clean $\mathrm{Au}(111)$ atom (red), $\mathrm{Au}(111)$ atom bound to $\mathrm{O}$ (black), and the difference between them (green-black); (c) $p$ projected electronic density of states (number of states per atom and per eV) of $\mathrm{O}$ on $\mathrm{Au}(111)$. Figures (d) and (e) display the same quantities but for the $\mathrm{Ag}$ case.

and electronic perturbation brought by $\mathrm{O}$ to the surface. The perturbation is particularly large for $\mathrm{Au}$, so the overall binding energy is significantly reduced and oxidation becomes unfavorable. Outstandingly, in terms of energy, the pure electronic relaxation caused by $\mathrm{O}$ adsorption is in turn a much more prominent perturbation than the geometric distortion: $E_{p e r t}$ is at least five times larger than $E_{r x}$ for $\mathrm{O}$ adsorption. And, while the latter assertions are so far inaccessible to experiment, we have substantiated them by contrasting Ag and Au with regard to (1) the distorted geometry of the chemisorbed surface, (2) the relaxation energy of such distortion $E_{r x}$, (3) the charge density redistribution (primarily depletion) occurring particularly along $\mathrm{Au}-\mathrm{Au}$ bonds upon $\mathrm{O}$ adsorption, (4) the energy associated to such charge density redistribution
$\left(E_{\text {pert }}-E_{r x}\right)$, and (5) a back analysis of the $a b$ initio binding energy via a multiple regression that determines by how much the covalent and ionic bonds increase it and by how much the surface perturbation decreases it, which is applicable to at least nine transition metals. We have thus provided for the first time a method to estimate the electronic and structural perturbation energies brought by adsorbates on surfaces.

We have also shown that attaching the nobility of gold to the increasing Pauli-repulsion orthogonalization terms with increasing $V_{s d}^{2}$ (see the Appendix) is not correct. If the binding of adsorbed species were increasingly weaker as one goes from $3 d$ to $5 d$ metals, the same trend should hold for metal-O dimers but it does not according to our results: the bond of the $\mathrm{O}-\mathrm{Au}$ dimer is stronger than that of the $\mathrm{O}-\mathrm{Ag}$ dimer. 
It must be acknowledged that denoting Au surfaces as noble or inert-in the sense that their surface atoms make weak bonds with oxidative agents - tells us that we have missed the relatively high readiness of $\mathrm{Au}$ to form covalent bonds with these. Au surfaces are not only not noble in this sense but less noble than $\mathrm{Ag}$ and $\mathrm{Cu}$; namely, among the three group-11 metals, the $d$-hybridization is strongest for Au. Moreover, we have shown that the $\mathrm{Au}-\mathrm{O}$ ionic bond is stronger than that made by $\mathrm{O}$ with many other transition metals. Thus, one can argue that $\mathrm{O}-\mathrm{Au}(111)$ is the least stable because of its deep $d$-band center, but only if compared to transition metals of earlier groups. One could also argue that $\mathrm{Au}$ is kept from corrosion because it sustains a relatively small charge transfer to $\mathrm{O}$, but this is true only with respect to elements of its own group, $\mathrm{Cu}$ and $\mathrm{Ag}$. However, $\mathrm{Au}$ stands among all nine investigated transition metals because it carries the largest energy cost for substrate perturbation, even though it is not a metal with particularly strong metal-metal bonds. We trace this not only to its deep $d$-band but also to the width of it, both features allow a particular hybridization between $\mathrm{O}-\mathrm{Au}$ bonding states at the expense of $\mathrm{Au}-\mathrm{Au}$ bonding states, as shown by our analysis of the electronic density of states of $\mathrm{Au}$ and $\mathrm{Ag}$ atoms in the bulk, at the clean surface layer, and bound to $\mathrm{O}$; namely, the electronic states populated to strengthen intralayer bonds in $\mathrm{Au}$ as the surface is created, are in an energy range that suits very well for hybridization with oxygen states but in such a way that $\mathrm{Au}-\mathrm{Au}$ bonding states are depleted.

The fact that not all substrates respond in the same manner to a given adsorbate and that the value of the binding energy mingles bond-strength and substrate perturbation hints that calculating reliably the local bond-strength of an adsorbate poses a challenge for measurements and calculations. This has critical implications because in recent times it has become common to use $E_{B}(\mathrm{O})$ as a descriptor for screening candidate catalysts, even though the catalytic activity depends on the binding of several other adsorbates. ${ }^{46,47}$ This approach assumes that the binding energy of all other adsorbates is a linear function of that of $\mathrm{O}$, and such assumption is in turn based on the $d$-band-center. ${ }^{46,47}$ Clearly, this approach does not consider whatsoever that the energy cost of the perturbation on the substrate caused by the various adsorbates will be quite different from one another. For example, the $\mathrm{Pt} / \mathrm{Au}(111)$ structure (proposed for ethanol oxidation ${ }^{48}$ ) undergoes such a reconstruction upon $\mathrm{OH}$ binding that the binding energy of $\mathrm{OH}$ is much weaker than predicted by the $d$-band center; namely, as seen from Table I of Ref. 30, the $d$-band center is much closer to the Fermi level for $\mathrm{Pt} / \mathrm{Au}(111)$ than for $\mathrm{Pt}(111)$ by $0.58 \mathrm{eV}$; yet, the binding energy of $\mathrm{OH}$ is only marginally higher than on $\operatorname{Pt}(111)$, it increases only by $0.04 \mathrm{eV}$. To contrast the poor performance of the $d$-band model, consider the case of $\mathrm{Pt} / \mathrm{Ru}(0001)$. The redshift of the d-band center with respect to $\mathrm{Pt}(111)$ is significantly smaller, $-0.37 \mathrm{eV}$, whereas the reduction of the binding energy of $\mathrm{OH}$ with respect to that on $\mathrm{Pt}(111)$ is significant, $-0.27 \mathrm{eV}$. Thus, we propose that $E_{\text {pert }}$ may help understand such binding energy trends. Then, since the binding energy of adsorbates is critical for understanding catalytic activity, we hope that our findings may contribute to the basis for the rational design of efficient catalysts and to go beyond bruteforce high-throughput computational screening.

\section{ACKNOWLEDGMENTS}

This work was supported the National Science Foundation under Grant No. CBET-1249134. The computations were performed in part at the Stokes Advanced Research Computing Center at the University of Central Florida.

\section{APPENDIX: DISCUSSION ON THE COVALENT AND IONIC OXYGEN-METAL BONDING}

\section{Modeling of $E_{d-h y b}$ and $E_{\text {ionic }}$}

Covalent binding. In modeling the $E_{d-h y b}$ contribution, we apply the expression proposed by Hammer and Nørskov ${ }^{17}$ within the framework of the tight-binding approximation (TBA) for the adsorption of CO. ${ }^{17}$ Such expression can and has been applied to describe the covalent bond between metal surfaces and other adsorbates (see, e.g., Ref. 23). A noteworthy aspect of the $d$-band-model formulation (and thus of the tightbinding framework) is that the importance of the HOMO and LUMO states of CO is not that they are molecular states but that one is unoccupied and the other one occupied. Of course, there is no splitting or HOMO-LUMO gap for atomic oxygen. Thus, if we put an oxygen atom in the system far enough from a metal surface to avoid a strong interaction with the metal, a single narrow peak of its $2 p$-states will be located at the Fermi-level $\left(\mathrm{E}_{F}\right)$ with four occupied states below $\mathrm{E}_{F}$ and two unoccupied states just above $\mathrm{E}_{F}$. These $\mathrm{O}$ states will hybridize with the metal $d$-states in the same way as those of a molecule with HOMO-LUMO gap do. Or, in the same way the occupied and unoccupied states of a gapless metal band, such as that of $\mathrm{Pt}$, interact with split molecular states.

For an arbitrary adsorbate, it can be written as follows:

$$
\begin{aligned}
E_{d-h y b} \simeq & -\left[f_{m} g_{u a} \frac{V_{o m \rightarrow u a}^{2}}{\epsilon_{u a}-\epsilon_{d}}-f_{m} g_{u a} S_{o m \rightarrow u a} V_{o m \rightarrow u a}\right] \\
- & {\left[\left(1-f_{m}\right) g_{o a} \frac{V_{o a \rightarrow u m}^{2}}{\epsilon_{d^{*}}-\epsilon_{o a}}\right.} \\
& \left.-\left(1+f_{m}\right) g_{o a} S_{o a \rightarrow u m} V_{o a \rightarrow u m}\right] .
\end{aligned}
$$

In the framework of the TBA, $E_{d-h y b}$ (Eq. (A1)) has four parts explicitly correlated: ${ }^{17}$ The first and second terms in brackets contain the interaction of occupied metal $d$-states (om) with unoccupied adsorbate states (ua). The third and fourth terms, in turn, represent the interaction of occupied adsorbate states (oa) with unoccupied $d$-metal states (um). The first and third terms account for bonding hybridization (gain of hybridization energy) and the second and fourth terms correspond to the orthogonalization energy cost. Thus, within the tight-binding-approximation framework, the first and third terms increase $E_{d-h y b}$ because they represent the hybridization between the metal $d$-states and the adsorbate $p$-states, whereas the second and fourth terms decrease $E_{d-h y b}$ because they represent the orthogonalization energy cost for the overlapping of these states. The latter is also known as Pauli repulsion because it is a repulsive interaction between metal 
and adsorbate that takes place when their non-orthogonal electronic states overlap, as required by Pauli exclusion principle.

$g_{o a}$ and $g_{u a}$ are the degeneracies of the occupied and unoccupied states of the adsorbate, respectively; $\epsilon_{o a}$ is the energy of the occupied states of the adsorbate with respect to $E_{F}$. Notice that there is also a contribution from the hybridization between the $s$-metal and $p$-adsorbate states but that is considered to vary little among transition metals. ${ }^{5,17}$

The occupancy of the bonding and anti-bonding states in this formulation is represented by $f_{m}=\frac{v-1}{10}$, which is the fractional filling factor of the $d$-band and $v$ is the valence of each metal surface atom. Note that even if $f_{m}=1$, only one part of the bonding hybridization energy (the donation from the adsorbate to the metal) in the d-band model

$$
-\left(1-f_{m}\right) g_{o a} \frac{V_{o a \rightarrow u m}^{2}}{\epsilon_{d^{*}}-\epsilon_{o a}}
$$

is exactly zero. Yet, the other part of the bonding hybridization energy (the back-donation from the metal to the adsorbate)

$$
-f_{m} g_{u a} \frac{V_{o m \rightarrow u a}^{2}}{\epsilon_{u a}-\epsilon_{d}}
$$

is not zero but maximized. Note also that all four terms in Eq. (A1) above are approximately proportional to the interatomic matrix element $V_{s d}^{2},{ }^{17,25}$

$$
\begin{gathered}
V_{o m \rightarrow u a}^{2} \approx \beta V_{s d}^{2}, \\
V_{o a \rightarrow u m}^{2} \approx \gamma^{2} \beta V_{s d}^{2}, \\
S_{o m \rightarrow u a} V_{o m \rightarrow u a} \approx \alpha \beta V_{s d}^{2},
\end{gathered}
$$

and

$$
S_{o a \rightarrow u m} \approx \alpha \gamma^{2} \beta V_{s d}^{2},
$$

where $\alpha, \beta$, and $\gamma$ are adjustable parameters to be determined via a multiple-variable regression. ${ }^{17}$ Note that, within the $d$-band model framework, these parameters are common to all metals for a given adsorbate. ${ }^{17}$ Thus, $E_{d-h y b}$ increases in magnitude with increasing extent of the $d$-orbitals because $V_{s d}^{2}$ increases with the extent of the d-states $\left(r_{d}\right),{ }^{25}$

$$
V_{s d}^{2} \propto r_{d}^{3} / a_{0}^{7}
$$

Then, by substituting Eqs. (A2) in (A1), one gets

$$
\begin{aligned}
E_{d-h y b} \simeq & -\beta V_{s d}^{2}\left[f_{m} g_{u a} \frac{1}{\epsilon_{u a}-\epsilon_{d}}-f_{m} g_{u a} \alpha\right. \\
& \left.+\left(1-f_{m}\right) g_{o a} \frac{\gamma^{2}}{\epsilon_{d^{*}}-\epsilon_{o a}}-\left(1+f_{m}\right) g_{o a} \alpha \gamma^{2}\right] .
\end{aligned}
$$

A fundamental aspect of the $d$-band model formulation (Eq. (A3)) is that the hybridization and the orthogonalization (Pauli repulsion) contributions are both proportional to $V_{s d}^{2} \cdot{ }^{16,17,49}$ Therefore, unless the term in squared brackets become negative for a given adsorbate and metal, the binding of the adsorbate is exothermic and the whole covalent part of the binding energy necessarily increases with increasing $V_{s d}^{2}$, i.e., with the extent of the metal $d$-states. Of course, if the term in squared brackets does becomes negative, then it means that the Pauli repulsion dominates and the covalent binding will become increasingly endoergic with increasing $V_{s d}^{2}$. The term in brackets of Eq. (A3) can be positive (exothermic binding) for group-11 metals because (1) the anti-bonding $d$-states of surface atoms are not fully occupied (metalmetal hybridization also takes place for group-11 metals, i.e., $\left.f_{m}<1\right)$. Thus, the fact that we have an analytic formulation of the $d$-band model (Eq. (A1)) and can estimate $f_{m}$ quite accurately from ab-initio calculations leaves no reason to simplify or take a priori that $f_{m}=1$ (i.e., all bonding and anti-bonding states are occupied), which strictly applies only for states of isolated atoms; (2) the repulsive orthogonalization terms contain the factor $\alpha$, where $\alpha \ll 1$; and (3) the $d$-band is not sufficiently deep, not even for Ag. For example, even if one assumes $f_{m}=1$ for group-11 metals, the $d$-band model applied to the weak chemisorption of $\mathrm{CO}$ on $\mathrm{Cu}(111)$ (see Eq. (1) of Ref. 17) yields a covalent-bond energy $E_{d-h y b}$ that contributes to the $\mathrm{CO}-\mathrm{Cu}(111)$ binding energy by $0.3 \mathrm{eV}$ (see Fig. 2 of Ref. 17). This shows that within the very $d$ band model formulation there is indeed an energy gain for hybridization despite $f_{m}=1$. The same study, on the other hand, finds that $\mathrm{CO}$ does not bind covalently to $\operatorname{Ag}(111)$ or $\mathrm{Au}(111)$ (Pauli repulsion dominates and the covalent binding is endoergic). Commonly, one would say that it is because both of these metals have larger $V_{s d}^{2}$ than $\mathrm{Cu}$ and thus stronger Pauli repulsion. However, that explanation does not justify the actual results: $V_{s d}^{2}$ is larger for $\mathrm{Au}$ than for $\mathrm{Ag}$, and yet the bond is more endoergic for $\mathrm{Ag}$ than for $\mathrm{Au}$. This shows that even if $f_{m}=1$, the dependence of $E_{d-h y b}$ on the $d$-band center remains through the interaction between occupied metal states and unoccupied adsorbate states. Moreover, ab-initio calculations of the charge density and density of states show that $\mathrm{CO}$ does chemisorb on $\mathrm{Ag}$ through the donation and backdonation (Blyholder ${ }^{50}$ ) mechanism, and this happens in part precisely because $f_{m} \neq 1$. $^{51}$

Ionic binding. In modeling the $E_{\text {ionic }}$ contribution, we consider an ideal ionic bond whose strength is determined by three input variables: (1) the metal-O charge exchange, which accounts for a binding-energy reduction because of charge removal from the metal atom (i.e., the work function) and a binding-energy increase because that charge is gained by $\mathrm{O}$ (electron affinity); (2) the binding-energy increase by the electrostatic metal-O attraction; and (3) the bindingenergy decrease because of the electrostatic metal-metal repulsion between the three metal atoms that lose electronic charge upon oxygen adsorption (see Eq. (A4)). Long-ranged interactions, periodicity, and the Pauli repulsive interaction between overlapping electrons clouds are assumed to be contained in the fitting parameters,

$$
\begin{aligned}
E_{\text {ionic }} \simeq & \frac{1}{4 \pi \epsilon_{0}}\left[-\lambda \frac{\Delta q_{O} \Delta q_{m}}{a_{0}}+\mu \frac{\Delta q_{m} \Delta q_{m}}{a_{0}}\right] \\
& +v\left(\Phi_{m}-E_{A}(O)\right) \Delta q_{O},
\end{aligned}
$$

where $\Delta q_{O}$ and $\Delta q_{m}$ are the charge gained by oxygen and that lost by the metal, respectively; $\Phi_{M}$ and $E_{A}(\mathrm{O})$ are the work function of the metal and the electron affinity of oxygen, respectively; and $\lambda, \mu$, and $v$ are adjustable parameters.

\section{More about the $d$-band model}

The $d$-band-center model originally aimed to correlate the binding energy of $\mathrm{CO}$ with the metal $d$-electronic states-the 
energy of the center of gravity of the $d$-band in particular. ${ }^{17}$ In this model, the $d$-band center $\epsilon_{d}$ typifies the contribution to the binding energy of $\mathrm{CO}$ that derives from the overlapping and hybridization among the electronic states of the metal surface and those of CO: the covalent-bond energy $E_{d-h y b}$. Importantly, as seen from the above Eq. (A1)-(A3), within the $d$-band model logic, bonds are expected to be stronger the closer the $d$-band center is to the Fermi level $E_{F}$ and the more extended the $d$-orbitals. ${ }^{17,18}$

Examples of inconsistencies between the d-band model predictions and experiment and/or ab initio calculations that involve group-10 and group-11 metals.

(a) Xin and Linic have reported that some adsorbates on $\mathrm{Pt}$ and Pd skin alloys do not follow the trends in adsorption energy predicted by the d-band model..$^{23}$

(b) $\mathrm{OH}$ binds covalently to all three metals: $\mathrm{Ag}, \mathrm{Cu}$, and Pt. Therefore, the Pauli-repulsion orthogonalization energy, in this case, is necessarily smaller than the hybridization energy for all of them, in smaller or larger extent depending on the metal. However, the contradiction appears when one realizes that $\mathrm{Pt}$ fulfills all the $d$-band model criteria to bind (covalently) $\mathrm{OH}$ stronger than $\mathrm{Ag}$ and $\mathrm{Cu}$, yet in reality Pt displays a weaker bonding. That is, the $d$-band center of $\mathrm{Pt}$ is closer to $E_{F}$ than those of $\mathrm{Ag}$ and $\mathrm{Cu}$. Moreover, Pt has a larger coupling matrix element and smaller fractional filling factor of the $d$-band than $\mathrm{Ag}$ and $\mathrm{Cu}$. Thus, by all considerations within the $d$-band model, $\mathrm{Pt}$ is predicted to bind $\mathrm{OH}$ stronger than $\mathrm{Ag}$ or $\mathrm{Cu}$. Therefore, the $d$-band model alone does fail to explain why $\mathrm{OH}$ binds stronger on Ag than on Pt. Even more so since exactly the opposite trend holds for atomic oxygen adsorption.

(c) $\mathrm{Cu}$ binds $\mathrm{O}$ stronger than $\mathrm{Au}$. However, because $r_{d}$ is relatively small for $\mathrm{Cu}-$ despite $a_{0}$ is smaller for $\mathrm{Cu}$ than for $\mathrm{Au}$ and the $d$-band center of $\mathrm{Cu}$ is closer to $E_{F}$ than that of $\mathrm{Au}$ - the interatomic matrix element $V_{s d}^{2}$ and $E_{d-h y b}$ are larger for $\mathrm{Au}$ than for $\mathrm{Cu}$.

(d) As shown in Fig. 1, $\epsilon_{d}$ for $\mathrm{Cu}$ is clearly below that for $\mathrm{Pt}$ and Pd. Thus, $\epsilon_{d}$ definitely cannot justify why the measured $H_{\text {ads }}^{D}$ of $\mathrm{O}$ on $\mathrm{Cu}(111)^{9}$ is larger than that on $\operatorname{Pt}(111)^{11,12}$ and even $\operatorname{Pd}(111)^{13}$ (nor can, of course, the interatomic matrix elements explain this case).

(e) It has been reported that despite a significant enhancement of the number of unoccupied antibonding states of $\mathrm{Pt} / \mathrm{Au}(111)$ with respect to $\mathrm{Pt}(111)$, the binding energy of $\mathrm{OH}$ is negligibly enhanced with respect to that on $\operatorname{Pt}(111){ }^{30}$

${ }^{1}$ H. Müller-Buschbaum, Z. Anorg. Allg. Chem. 628, 2559 (2002).

${ }^{2}$ P. Pyykkö, Angew. Chem., Int. Ed. 43, 4412-4456 (2004).

${ }^{3}$ M. Haruta, Catal. Today 36, 153 (1997).

${ }^{4}$ S. Stolbov and M. Alcántara Ortigoza, J. Phys. Chem. Lett. 3, 463 (2012), and references therein.

${ }^{5}$ H. Over, Physics of Covered Solid Surfaces: Adsorbed Layers on Surfaces. Section 3.4.2 (Landolt Börnstein, Springer-Verlag, Berlin, Heidelberg, 2005), and references therein.

${ }^{6}$ E. German and I. Efremenko, J. Mol. Struct.: THEOCHEM 711, 159 (2004), and references therein.

${ }^{7}$ C. T. Campbell, Surf. Sci. 157, 43 (1985).

${ }^{8}$ N. Saliba, D. Parker, and B. Koel, Surf. Sci. 410, 270 (1998)

${ }^{9}$ R. M. Dell, F. S. Stone, and P. F. Tiley, Trans. Faraday Soc. 49, 195 (1953).

${ }^{10}$ A. Raukema, D. A. Butler, F. M. Box, and A. W. Kleyn, Surf. Sci. 347, 151 (1996).
${ }^{11}$ Y. Y. Yeo, L. Vattuone, and D. A. King, J. Chem. Phys. 106, 392 (1997).

${ }^{12}$ T. Matsushima, Surf. Sci. 157, 297 (1985).

${ }^{13}$ G. Zheng and E. Altman, Surf. Sci. 462, 151 (2000).

${ }^{14}$ C. Stampfl, H. J. Kreuzer, S. H. Payne, H. Pfnür, and M. Scheffler, Phys. Rev. Lett. 83, 2993 (1999).

${ }^{15} \mathrm{H}$. Engelhardt and D. Menzel, Surf. Sci. 57, 591 (1976).

${ }^{16}$ B. Hammer and J. K. Nørskov, Nature 376, 238 (1995).

${ }^{17}$ B. Hammer, Y. Morikawa, and J. K. Nørskov, Phys. Rev. Lett. 76, 2141 (1996).

${ }^{18}$ This approximation relies on the dominance of the term accounting for the hybridization between occupied metal $d$-states with unoccupied adsorbate states in $E_{d-h y b}$, because such term is inversely proportional to $\epsilon_{u a}-\epsilon_{d}$ (see the Appendix), where $\epsilon_{u a}$ is the energy of the unoccupied states of the adsorbate and both, $\epsilon_{u a}(>0)$ and $\epsilon_{d}(<0)$, are with respect to $E_{F}$.

${ }^{19}$ J. P. Desclaux and P. Pyykkö, Chem. Phys. Lett. 39, 300-303 (1976).

${ }^{20}$ J. R. Kitchin, J. K. Nørskov, M. A. Barteau, and J. G. Chen, J. Chem. Phys. 120, 10240 (2004)

${ }^{21}$ J. Zhang, M. B. Vukmirovic, Y. Xu, M. Mavrikakis, and R. R. Adzic, Angew. Chem. 117, 2170-2173 (2005).

${ }^{22}$ J. K. Nørskov, T. Bligaard, J. Rossmeisl, and J. Rossmeisl, Nat. Chem. 1, 39 (2009), and references therein.

${ }^{23}$ H. Xin and S. Linic, J. Chem. Phys. 132, 221101 (2010).

${ }^{24}$ H. A. Hansen, J. Rossmeisl, and J. K. Nørskov, Phys. Chem. Chem. Phys. 10, 3722 (2008)

${ }^{25}$ W. A. Harrison, Electronic Structure and the Properties of Solids: The Physics of the Chemical Bond (W. H. Freeman and Company, 1980).

${ }^{26}$ S. Nishimura, A. T. N. Dao, D. Mott, K. Ebitani, and S. Maenosono, J. Phys. Chem. C 116, 4511-4516 (2012).

${ }^{27}$ M. A. MacDonald, P. Zhang, N. Chen, H. Qian, and R. Jin, J. Phys. Chem. C 115, 65-69 (2011).

${ }^{28}$ F. Liu, D. Wechsler, and P. Zhang, Chem. Phys. Lett. 461, 254 (2008).

${ }^{29}$ D. M. Mott, D. T. N. Anh, P. Singh, C. Shankar, and S. Maenosono, Adv. Colloid Interface Sci. 185-186, 14 (2012).

${ }^{30}$ S. Stolbov and S. Zuluaga, J. Phys. Chem. Lett. 4, 1537 (2013).

${ }^{31}$ G. Kresse and J. Furthmüller, Comput. Mater. Sci. 6, 15 (1996).

${ }^{32} \mathrm{G}$. Kresse and J. Joubert, Phys. Rev. B 59, 1758 (1999).

${ }^{33}$ M. Payne, M. Teter, D. Allan, T. Arias, and J. Joannopoulos, Rev. Mod. Phys. 64, 1045 (1992).

${ }^{34}$ J. P. Perdew, K. Burke, and M. Ernzerhof, Phys. Rev. Lett. 77, 3865 (1996).

${ }^{35}$ Y. Wang, N. S. Hush, and J. R. Reimers, Phys. Rev. B 75, 233416 (2007).

${ }^{36}$ H. J. Monkhorst and J. D. Pack, Phys. Rev. B 13, 5188 (1976).

${ }^{37}$ R. F. W. Bader, Atoms in Molecules: A Quantum Theory (Oxford University Press, New York, 1994).

${ }^{38}$ A. Kokalj, Comput. Mater. Sci. 28, 155 (2003), code available from http:// www.xcrysden.org/.

${ }^{39}$ Despite of the $\sim 1 / \mathrm{r}$ interaction-energy dependence of the Coulomb potential of the interaction between $\mathrm{O}$ and the metal atoms, the potential is fairly harmonic (symmetric upon displacements in three orthogonal directions) for displacements of up to $0.03 \AA$, well beyond the atomic displacements used to obtain the force constants.

${ }^{40}$ A. Haaland, Molecules and Models: The Molecular Structures of Main Group Element Compounds (Oxford University Press, 2008).

${ }^{41}$ Ionic bonds often render softer force constants than covalent bonds even in cases in which the ionic bond is stronger than the covalent one. ${ }^{40}$ This is because the strength of the ionic bond decays as $\sim 1 / \mathrm{r}$ while the covalent bond has a much shorter range since, at least withing the tight-bonding approximation, the overlapping wavefunctions decay exponentially.

${ }^{42}$ J. Autschbach, J. Chem. Phys. 136, 150902 (2012).

${ }^{43}$ M. Alcantara Ortigoza and S. Stolbov, APS March Meeting Abstracts, 2013 p. G5013, URL: http://adsabs.harvard.edu/abs/2013APS..MAR. G5013A.

${ }^{44}$ C.-Y. Lu and G. Henkelman, J. Phys. Chem. Lett. 2, 1237 (2011)

${ }^{45} \mathrm{Au}$ displays a very small charge density accumulation that is not present in Ag. This one, however, is basically localized around Au atoms and thus does not contributing to the bonding.

${ }^{46}$ J. Greeley, I. E. L. Stephens, A. S. Bondarenko, T. P. Johansson, H. A. Hansen, T. F. Jaramillo, J. Rossmeisl, I. Chorkendorff, and J. K. Nørskov, Nat. Chem. 1, 552 (2009).

${ }^{47}$ J. Greeley and J. K. Nørskov, J. Phys. Chem. C 113, 4932-4939 (2009).

${ }^{48}$ M. Li, P. Liu, and R. R. Adzic, J. Phys. Chem. Lett. 3, 3480 (2012).

${ }^{49}$ F. Abild-Pedersen, J. Greeley, F. Studt, J. Rossmeisl, T. Munter, P. Moses, E. Skúlason, T. Bligaard, and J. Nørskov, Phys. Rev. Lett. 99, 016105 (2007).

${ }^{50} \mathrm{G}$. Blyholder, J. Phys. Chem. 68, 2772 (1964).

${ }^{51}$ M. Alcántara Ortigoza, R. Heid, K. P. Bohnen, and T. S. Rahman, J. Phys. Chem. A 115, 7291-7299 (2011). 